\title{
De l'Europe politique en particulier et de l'anthropologie en général
}

\section{Marc Abélès}

\section{(2) OpenEdition}

1 Journals

\section{Édition électronique}

URL : http://journals.openedition.org/conflits/379

DOI : $10.4000 /$ conflits.379

ISSN : $1777-5345$

Éditeur :

CCLS - Centre d'études sur les conflits lilberté et sécurité, L'Harmattan

\section{Édition imprimée}

Date de publication : 15 janvier 1997

ISSN : 1157-996X

\section{Référence électronique}

Marc Abélès, « De l'Europe politique en particulier et de l'anthropologie en général », Cultures \& Conflits [En ligne], 28 | hiver 1997, mis en ligne le 07 mars 2006, consulté le 30 mars 2021. URL : http:// journals.openedition.org/conflits/379; DOI : https://doi.org/10.4000/conflits.379

Ce document a été généré automatiquement le 30 mars 2021.

Creative Commons License 


\title{
De l'Europe politique en particulier et de l'anthropologie en général
}

\author{
Marc Abélès
}

Marc ABELES ${ }^{1}$

Prenant un peu de recul par rapport aux recherches que j'ai menées sur le Parlement et la Commission européens, je souhaiterais proposer ici quelques pistes de réflexion concernant l'approche d'une réalité politique complexe dont la compréhension requiert les efforts conjugués de plusieurs disciplines. Comme je l'ai souvent indiqué, cette complémentarité des regards est une condition essentielle pour une meilleure connaissance du système communautaire. Cette dernière expression pose cependant un problème : y a-t-il à proprement parler un système communautaire ? Faut-il à tout prix chercher une cohérence dans un tissu d'institutions qui ont connu un développement inégal et ont été construites dans le cadre de rapports de force et de négociations entre des gouvernements nationaux? Peut-on identifier un objet qu'un des plus pertinents protagonistes de l'Europe a lui-même désigné comme " objet non identifié "? Au chercheur, la construction communautaire pose un intéressant problème, celui d'un modèle d'incertitude qui n'est nullement incompatible avec l'existence d'institutions et de politiques dont la solidité apparaît incontestable. Aussi bien, peut-on aisément ignorer l'inachèvement de l'Europe et la traiter comme s'il s'agissait d'un système politique réalisé. Cette position théorique présente l'avantage de susciter nombre d'analyses indispensables des procédures et des modes d'action qui caractérisent la gouvernance européenne. Le regard de l'anthropologue Mon point de vue d'anthropologue est un peu différent: ce qui m'intéresse, c'est la co-présence permanente dans la sphère communautaire de l'actualité immédiate et du projet non encore réalisé. Pour parler un langage aristotélicien, l'Europe est tout à la fois en acte et en puissance. Ce mélange de quotidien et d'anticipation constitue une des spécificités de cet univers. A dessein, je souligne cette dimension du temps, car il me semble que l'Union entretient avec l'histoire une relation complexe et ambiguë. L'Europe représente curieusement ce qui nous est le plus contemporain et une sorte de planète éloignée que les citoyens scrutent avec appréhension (parfois avec 
résignation). En ce qui concerne le chercheur, le travail d'analyse des mécanismes européens vise à limiter cette distance planétaire. Mieux connaître, c'est nous permettre, sinon de nous approprier, du moins de nous familiariser avec les contraintes dans lesquelles nous vivons désormais et qui ont nom globalisation, compétition économique mondiale, primauté des relations politiques internationales, etc. Il y a là un effort de portée pédagogique réelle : la science sociale participe ici à une entreprise de familiarisation dont on ne saurait nier l'utilité. Ce projet contraste cependant avec la perspective dans laquelle je me situe ${ }^{2}$ : celle-ci privilégie la distance et favorise une démarche axée sur ce que les anthropologues américains nomment " defamiliarization ". Cette investigation vise à prendre au sérieux la singularité du processus communautaire, en l'abordant sous des angles parfois incongrus. Pour ce faire, on a recours à l'observation minutieuse des comportements des acteurs et à une écoute attentive des discours qu'ils tiennent à propos de leur pratique quotidienne de l'institution. J'ai opposé cette mise en perspective " par le bas ", à celles, plus répandues, qui privilégient la cohérence du système et ses fonctionnalités. On ne s'étonnera pas que deux séries de données aient plus particulièrement retenu l'attention de l'anthropologue: d'une part, le phénomène de déterritorialisation et le changement d'échelle qui s'opèrent dans le travail communautaire; d'autre part, la réalité multiculturelle de cet univers. Il y a là une conjonction entre deux états de fait qui, pris séparément, n'auraient sans doute pas le même impact : imbriqués de la sorte, ils impriment à l'action une dynamique et des tensions spécifiques. Je ne reviendrai pas ici sur les principaux résultats de notre exploration de cet alliage complexe entre compromis culturel et culture politique du compromis3. Me tournant vers l'anthropologie, je souhaiterais m'interroger sur la place de ces recherches dans le développement de la discipline. Personne, il y a vingt ans, n'aurait pensé que l'Europe politique pût intéresser de près ou de loin les anthropologues. Qu'on développe des recherches ethnographiques sur le monde rural, qu'on s'intéresse aux minorités porteuses de traditions ou au quotidien des quartiers dans les villes, il y avait là un domaine d'extension de l'anthropologie hors de l'exotisme qui pouvait paraître légitime. Un mot magique connotait assez bien le champ de phénomènes réservés à la discipline: l'identité. Ou plutôt d'ailleurs, le couple identité/altérité: le " regard éloigné " sur l'Autre devait permettre d'en mieux saisir les traits identitaires. Au début des années quatre-vingt, quand j'entreprenais d'étudier l'univers des élus locaux en France $^{3}$, ce projet était interprété comme une exploration du terroir et de pratiques considérées comme en voie de disparition. L'anthropologie du contemporain s'apparentait ici à un relevé des archaïsmes et des pesanteurs qui grevaient encore la vie politique française. Pour les politistes comme pour les anthropologues, la saveur de ces histoires de famille dont j'ai émaillé ma recherche avait quelque chose de rassurant. L'" objet " correspondait bien à ce qu'on attendait d'une production ethnographique. L'analyse des liens de parenté, des rituels et des symboles, le souci du lieu et de la durée, légitimaient en quelque sorte une extension du champ de l'anthropologie à un domaine jusqu'alors peu fréquenté. En même temps, cette approche suscitait une série de questions relatives aux cadres spatial et temporel dans lesquels elle se déployait et à la position du chercheur dans ce contexte. Ce qui s'opérait dans ce travail, c'était un vaet-vient: au plan spatial, varier la focale du micro-local au national, était le moyen d'approfondir la connaissance des élus. Au plan temporel, ce qui retenait mon attention, c'était la superposition au présent des traces réelles ou symboliques du passé. Sans revenir ici sur les avancées et les limites de la recherche, je retiendrai 
surtout le souci de ne pas réifier l'objet ethnographique, ces élus du terroir, en leur conférant une " identité ", en l'occurrence celle d'incarnations d'un " système républicain " finissant. Comme les faits l'ont montré par la suite, ils représentaient une réalité bien vivace, renforcée par la décentralisation. La " modernisation " ne s'est pas produite, ou du moins pas sur le mode d'une brutale substitution d'un modèle d'organisation homogène et prégnant, ainsi qu'avaient pu le prédire les observateurs. En fait, ce type d'interprétation pose deux problèmes : l'un concerne la propension à réifier les identités ; l'autre a trait au partage qui s'opère entre tradition et modernité. Comment faire une anthropologie au présent sans être sujet à la tentation du " faux archaïsme " ou, à l'inverse, tomber dans le piège d'une description superficielle de l'actualité? Le jeu mouvant des altérités Pour revenir au premier problème posé, constatons que la recherche sur la construction européenne rejoint une interrogation plus générale des anthropologues du contemporain sur les présupposés qui commandent la production des données et le texte ethnographique, qui les incite à remettre en question la notion même d'altérité. L'espace de l'anthropologie a longtemps été circonscrit par une dialectique subtile entre identité et altérité. A défaut d'avoir en face de soi un Autre lointain et exotique, on s'employait à mettre à distance le proche (individu ou groupe) pour en mieux faire émerger ce qui constitue " son " identité. La technique n'est pas neuve, c'est celle du Montesquieu des Lettres Persanes. Son efficacité n'est plus à vanter, et nous ne nous privons pas de jouer au Persan sur le terrain : l'ethnographie offre un tableau original et souvent inattendu d'un quotidien auquel nous sommes trop englués pour en discerner les contours. N'est-il pas temps, non seulement de dépasser une opposition plus ou moins rigide entre le soi et l'Autre, entre le sujet et l'objet, mais de repenser ce qu'on pourrait appeler les " régimes d'altérité " ? Une des évolutions positives de l'anthropologie est sans conteste d'avoir désubstantifié l'Autre: ce qui pouvait apparaître comme une réalité, un donné élémentaire, nous l'appréhendons aujourd'hui comme une construction dont il s'agit de mieux rendre compte. Le développement d'une anthropologie du proche n'a fait que compliquer un peu plus les choses: " le lieu de l'altérité s'est déplacé et en quelque sorte intériorisé "4. En se rapprochant géographiquement de son objet, le chercheur découvre qu'il n'a pas affaire à de l'identique : la familiarité n'est jamais au rendezvous. Cette constatation ne saurait cependant en elle-même suffire à satisfaire nos exigences épistémologiques. Il vaut aussi la peine de se demander si l'anthropologie n'a pas tout à gagner à produire de nouveaux lieux d'investigation qui offriraient une perspective heuristique nous permettant de sortir du cercle enchanté où nous enferme la dialectique de l'Autre et de l'identique. L'approche de la construction européenne qui met en scène le jeu mouvant des altérités dans une entreprise focalisée sur l'édification d'une communauté, peut être définie comme une entreprise de " défamiliarisation ". Je reprends à dessein l'expression employée par les anthropologues américains Marcus et Fischer ${ }^{5}$, car elle indique bien l'un des apports du travail ethnographique. Qu'entendre par " défamiliarisation " ? Au premier abord, on pourrait penser qu'il s'agit d'une mise à distance: faire comme si ces praticiens de l'Europe étaient une tribu lointaine, avec ses mœurs, ses codes, ses rites. " Faire comme si ", c'est-à-dire mimer le rapport canonique entre l'anthropologue et son Autre. Ce serait retomber dans l'illusion du regard éloigné, métaphore qui porte en elle l'idée d'une transparence possible, d'un accès possible aux ressorts de cet Autre, une fois qu'on a trouvé la bonne focale. La défamiliarisation constitue une opération différente : elle met en scène le jeu des altérités au cœur de l'identique. C'est ainsi que l'entité 
ethnographique, " le " fonctionnaire européen se fragmente de par sa réinscription dans un espace de confrontations où l'on met en évidence l'incongruité des points de vue, les décalages et les malentendus (misunderstanding, mismatch) qui s'ensuivent. Cette fragmentation, loin d'être synonyme d'une dilution de l'objet dans les méandres interminables de la recherche, est un moment indispensable d'un patient travail qui est aux antipodes d'une entreprise de dévoilement, où la " lumière naturelle " de l'entendement guiderait le chercheur? C'est la référence leibnizienne à la " connaissance aveugle ou symbolique " qui paraît la plus pertinente pour caractériser le type de rationalité qui prévaut dans l'investigation. L'anthropologie et la question du temps J'en viens au second problème, celui du temps et de l'historicité. Abordant les institutions européennes, l'anthropologue se trouve projeté dans un processus de fabrication " en temps réel ", mais il a affaire à des individus qui conçoivent leur action en regard d'un avenir qui constitue la toile de fond du présent. Comment travailler au présent sur du présent? Est-il possible d'analyser " en temps réel " des processus dont on perçoit bien qu'ils affectent nos sociétés, mais dont il est difficile de mesurer les effets, sauf de manière très superficielle? Mais surtout, comment réfléchir notre rapport au temps, aussi bien du point de vue de l'observateur que de celui de l'observé, dans un monde qui semble perplexe par rapport à sa propre capacité d'innovation? La question du devenir et de ses représentations hante les ethnologues autant que les historiens. C. Lévi-Strauss, en introduisant la célèbre distinction entre " sociétés froides " et " sociétés chaudes ", répartissait les tâches entre les ethnologues spécialistes des premières et leurs collègues sociologues et historiens chargés de l'investigation des secondes. Le caractère tranché de cette opposition suscita un vaste débat dont la polémique entre Sartre et Lévi-Strauss reflète bien l'enjeu. En fait, deux questions différentes étaient posées : d'une part, l'anthropologie, en mettant l'accent sur l'inertie des structures, ne se condamnait-elle pas à ignorer la " praxis ", c'est-à-dire la dynamique même de la société ? D'autre part, la détermination d'un domaine privilégié de l'anthropologie, les sociétés froides, ne découlait-elle pas de cette fascination pour les structures ? Lévi-Strauss devait se défendre sur un double front : il lui était reproché non seulement de réduire le social à un système de contrainte, réduisant à néant l'initiative et la liberté humaine, mais aussi de refuser l'omniprésence de l'histoire d'un bout à l'autre de la planète. A relire aujourd'hui les textes de l'auteur de Tristes tropiques et de ses contradicteurs, on aperçoit que ce qui choque ces derniers, c'est le fait que les " sauvages " puissent échapper, non point à l'histoire, mais à une philosophie de l'histoire, elle-même datée, et qui, avec le recul, peut sembler désuète. Mais au début des années soixante, la simple hypothèse que ces sociétés ne ressortissent pas de la philosophie de l'histoire, en se logeant parmi les modes de production successivement engendrés par l'humanité, avait de quoi apparaître comme démobilisatrice. Cela équivalait à considérer l'histoire, telle que la vivent et la pensent nos contemporains, comme une modalité parmi d'autres possibles du devenir humain? Quid du progrès, quid des luttes séculaires des opprimés, face à ce relativisme exacerbé ? Or, curieusement, Lévi-Strauss n'a, de son côté, jamais cessé de répéter que l'histoire est partout. Comme il l'explique dans sa Leçon inaugurale de 1960, coupant en quelque sorte l'herbe sous le pied à ses adversaires: " Bien sûr les sociétés dites primitives sont dans l'histoire; leur passé est aussi ancien que le nôtre puisqu'il remonte aux origines de l'espèce " ${ }^{6}$. L'anthropologue est avant tout sensible à la perception du devenir telle qu'elle se manifeste chez les membres des groupes étudiés. En particulier, c'est l'absence d'un temps continu, progressif, chargé d'innovation, qui 
lui paraît cohérent avec la place impartie aux mythes dans la pensée sauvage. Cela amène tout naturellement Lévi-Strauss à distinguer ce " temps mécanique et réversible " à notre perception d'un " temps statique et irréversible "7. Le temps " moderne ", si l'on ose dire, se situerait dans l'horizon du progrès, par rapport à une vision plus axée sur l'origine et télescopant présent et passé. Que les sociétés dites primitives ne semblent pas éprouver, comme les nôtres, le souci du futur, on ne cesse de le constater en se plongeant dans la littérature ethnographique. Lévi-Strauss insiste, en outre, sur le fait que leur " sagesse particulière... les incitent à résister désespérément à toute modification de leur structure qui permettrait à l'histoire de faire irruption dans leur sein "8. Cette allergie à la modernité semble fasciner l'anthropologue qui, au vu des dégâts suscités par " l'irruption " d'une histoire écrite par l'Occident, peut légitimement s'interroger sur les bienfaits de ce qu'on a coutume de désigner par le mot " progrès ". On a parfois reproché son pessimisme à Lévi-Strauss : cela revenait à lui faire grief de la fascination que peut éprouver l'ethnologue à l'égard d'un usage du temps différent du nôtre. Ce qui nous importe, en relisant ces textes, c'est le repérage qu'ils opèrent d'une forme d'historicité spécifique. Ce faisant, ils nous incitent à réinterroger notre propre régime d'historicité, c'est-à-dire la relation que nous entretenons avec le temps. Certes on a pu taxer la position lévi-straussienne d'idéaliste, voire même de conservatrice, mais on peut tout aussi bien voir dans ce souci de donner la parole à l'Autre, l'instrument d'une critique efficace de la vision de l'histoire dominante dans les sciences sociales. Pour s'en convaincre, il suffit a contrario de constater avec quelle sérénité Lévi-Strauss évacue toute possibilité de conflit entre l'histoire et l'anthropologie, répartissant ainsi les domaines de compétences: " l'une déploie l'éventail des sociétés humaines dans le temps, l'autre dans l'espace "9. L'affaire est entendue, mais ce qui ne peut qu'agacer les historiens, c'est de voir quelques pages plus loin, l'anthropologie objectiver leur conception du temps et en démonter les codes à la manière dont elle traiterait la pensée mythique. On voit bien qu'au-delà des conflits de territoires entre disciplines connexes, le véritable enjeu concerne l'existence ou non de différents modes d'être au temps, qui, à leur tour, conditionnent les manières de faire l'histoire. Il faut reconnaître que la discussion ouverte par Lévi-Strauss a été progressivement recouverte par un débat axé sur l'opposition entre structure et histoire : les deux perspectives étaient-elles conciliables, l'une des deux orientations devait-elle se subsumer à l'autre? Les anthropologues qui sont soucieux de ne pas perdre de vue l'immersion " dans l'histoire " des sociétés qu'ils étudient s'accommodent difficilement de la tendance du structuralisme à mettre l'accent sur les discontinuités et à opposer sociétés " froides " et sociétés " chaudes ". L'anthropologie saisie par l'histoire Deux voies différentes ont été empruntées par ceux qui ont choisi d'historiciser le champ de l'anthropologie. Dès 1955, G. Balandier dans Sociologie actuelle de l'Afrique noire met l'accent sur l'importance des dynamiques sociales et amorce une longue série de recherches où la thématique du conflit, du changement et de l'innovation joue un rôle essentiel. L'histoire est omniprésente, même dans des sociétés qui peuvent paraître avoir échappé à l'emprise du temps. Mais aucune d'entre elles n'échappe à " l'illusion sociale essentielle ", que Balandier résume ainsi : " Toute société se saisit moins sous l'aspect de ce qu'elle est: un perpétuel procès d'engendrement, que sous l'aspect d'un ordre établi, durable ${ }^{10}$. Certaines sociétés ont tendance à se complaire dans la conservation du passé, et celles-ci méritent l'appellation de " sociétés traditionnelles ". Le monde de l'anthropologue se trouve en quelque sorte scindé en deux : il s'ordonne autour du couple tradition/modernité, et 
c'est le second pôle du couple qui intéresse plus directement Balandier : on notera au passage comment au fil de ses publications, l'expression " sociologie actuelle " s'est effacée devant celles d'" anthropologie " et de " modernité ". La question du pouvoir, des tensions et des changements qu'il engendre, est au cœur de cette interrogation qui se présente comme une perspective dynamiste sur les sociétés. Dans le prolongement des débats autour de l'histoire et de la structure, le néo-marxisme des années soixantedix représente une manière différente de réintroduire l'histoire, tout en faisant son miel des principaux acquis de l'anthropologie structurale. Certes, on critique la sousestimation du " continent histoire " par le structuralisme. Cependant, on en reconnait tout l'apport, en regard des dérives empiristes des sciences humaines, au point que l'un des principaux instruments utilisés par Althusser pour penser les rapports entre les différents niveaux du social est le concept de " causalité structurale ". Comment penser la dominance d'un niveau sur les autres? Comment rendre compte des contradictions entre structures, y compris internes à l'une d'entre elles? Concernant les positions de Lévi-Strauss, M. Godelier s'emploie à discerner l'élément idéaliste et les convergences entre les préoccupations de l'auteur de La Pensée sauvage et celles de Marx ${ }^{11}$. Paraphrasant une inscription de l'époque, tout se passe comme si le structuralisme était soluble dans le matérialisme historique. E. Terray considère que l'anthropologie n'est autre qu'une province de cette science de l'histoire ${ }^{12}$. Ce qui frappe rétrospectivement, c'est l'effort entrepris, moins pour réconcilier les deux approches que pour intégrer le point de vue lévi-straussien dans une vision continuiste du devenir humain. Certes, on est loin de l'évolutionnisme à la Morgan. Les auteurs se réclamant du marxisme insistent sur le caractère plurilinéaire de l'évolution. Mais la question de l'être au temps, d'univers différents du nôtre, semble étrangère à cette perspective. Ce qui importe, " en dernière instance ", ce sont les systèmes et leurs contradictions : la notion de modernité n'a guère de sens, et l'on raisonne sur des logiques globales, qu'il s'agit de reconstituer en-deçà des actions produites par les sujets. Ces derniers se meuvent dans l'illusion: les discours sur la modernité comme ceux qui exaltent la tradition relèvent de l'idéologie, de l'obscur et du confus. Dans cette perspective, le changement relève d'une machinerie englobante, d'un " procès sans sujet ". On a affaire à une historicisation hors-temps dans un monde d'instances, de niveaux, de fonctions, et l'objet de l'anthropologie est identifié à un type bien particulier de mode de production " précapitaliste ". Historicisation, certes, puisque l'objet de l'anthropologue est inscrit dans la succession des époques de l'humanité ; hors temps, néanmoins, car l'étude des temporalités différentielles, agies ou subies par les protagonistes, n'intéresse pas directement cette approche. C'est un véritable jeu de chassés-croisés qui caractérise les relations entre histoire et anthropologie. Le structuralisme jette un pavé dans la mare, en introduisant à titre d'hypothèse de travail l'idée de sociétés à degré zéro d'histoire; de quoi faire frémir les tenants du matérialisme. Mais ce faisant, Lévi-Strauss se préoccupe de rendre compte de la différence que manifestent les sociétés froides et les sociétés chaudes dans " leur attitude envers leur passé respectif "13. A l'inverse, les chercheurs néo-marxistes veulent réhistoriciser le social, mais se soucient peu d'explorer la dimension temporelle $\mathrm{du}$ social. La grande affaire de ces analyses est l'articulation des instances ou niveaux, et l'importance accordée à la métaphore topique est révélatrice. Il n'en reste pas moins que l'insistance sur l'historicité des sociétés ouvre un champ de questions dont $\mathrm{S}$. Ortner, dans l'étude qu'elle a consacrée aux théories anthropologiques depuis les années soixante ${ }^{14}$, montre bien la nouveauté. Selon elle, la recherche des sixties était 
dominée par trois approches: l'anthropologie symbolique dominée par Geertz et sa problématique de la culture comme texte, de l'autre par Turner et son intérêt pour la dimension pragmatique des symboles, et par l'écologie culturelle (Harris, Rappoport) et son usage du concept d'adaptation pour construire une théorie matérialiste de la culture ; le structuralisme, jamais vraiment populaire aux Etats-Unis, mais adopté par les Britanniques et qui devint leur version à eux de l'anthropologie symbolique, une théorie de la superstructure. Cette situation s'est trouvée modifiée par la vogue du marxisme dans les années soixante-dix. Le débat sur l'historicité des sociétés ouvre un nouvel axe de recherche qui va devenir prépondérant durant la décennie. Un concept apparaît central, celui de pratique. Déjà présente dans des approches telles que l'interactionnisme symbolique en sociologie ou le transactionnalisme en anthropologie, la thématique de l'action et des relations entre système et pratique s'affirme dans les recherches de Bourdieu ${ }^{15}$ et de Sahlins ${ }^{16}$. Stratégies; habitus; intérêts; tous ces concepts participent d'une réflexion où le changement est envisagé en prenant en compte les acteurs et leurs projets. Les théoriciens de la pratique jouent, d'une double référence, à Marx et à la praxis, mais aussi à Weber et à l'acteur. La périodisation proposée par Ortner peut apparaître simplificatrice sous certains aspects. Mais, pour l'essentiel, elle a le mérite de marquer comment la conjoncture théorique s'est radicalement transformée à partir des années quatre-vingt. Si le marxisme présentait, et parfois jusqu'à la caricature, une tendance à accentuer le système au détriment de la pratique, qui l'apparentait au structuralisme, il n'en ouvrait pas moins un espace d'investigation critique sur le devenir des sociétés. Le recentrage sur l'action viendra une décennie plus tard. Déjà, l'un des principaux " structuralo-marxistes ", Godelier, en introduisant comme thème de recherche majeure la question de la transition, était amené à s'interroger sur les décalages entre processus relevant de modes de production différents. L'on voit alors se dessiner en pointillé la possibilité d'une problématique prenant en compte la différence des temps au sein des pratiques. Le retour du contemporain On remarquera que dans le contexte de la réintroduction de la praxis en anthropologie, les questions concernant l'" actualité " et la " modernité ", qui traversaient l'approche dynamiste, trouvent une pertinence nouvelle. L'observateur est amené à réfléchir sur la manière dont il appréhende l'action des individus qu'il observe, et sur ses propres anticipations dans le processus de construction d'un compte rendu ethnographique. On peut mettre en parallèle comme l'a fait A. Bensa ${ }^{17}$ cette démarche et celle des micro-historiens, et leur souci de restituer " les comportements concrets dans toute leur complexité ambiguë car ils se réfèrent à des normes multiples et contradictoires "18. A la différence du modèle dominant de l'histoire sociale qui privilégiait le global et la durée, les micro-historiens s'intéressent tout particulièrement au local et aux stratégies individuelles, et tentent de reconstruire le " système de contextes "19 dans lesquels se meuvent les acteurs. Peut-il en être autrement pour un anthropologue qui, lui, partage le présent du groupe sur lequel il travaille ? Or, comme l'ont fait remarquer des anthropologues américains tels que Fabian ${ }^{20}$, Marcus et Fischer ${ }^{21}$, les textes ethnographiques ont toujours tendu à oblitérer la présence des sujets concrets auxquels ils ont affaire. " Alors que la micro-histoire donne accès à la présence passée du temps, l'anthropologie s'installe dans un éternel présent ", écrit à juste titre Bensa ${ }^{22}$. C'est dans le contexte d'une critique des conceptions culturalistes et fonctionnalistes que Fabian vise notamment à réintroduire le thème du temps. Critiquant l'hégémonie d'un mode de représentation qui met l'objet de l'ethnologue à distance, il préconise la mise en œuvre d'une " processual and materialist theory ". Les 
anthropologues ont été incapables de penser la contemporanéité (coevalness) de l'Autre. Cette observation est, selon lui, aussi valable pour les marxistes que pour " the bourgeois positivist anthropology " ${ }^{23}$. Il ne suffit pas d'invoquer le primat de l'histoire sur la structure ou la dynamique du changement : on n'évite pas l'allochronisme, cette manière de mettre l'Autre à l'écart de notre temps, en neutralisant un élément essentiel qui réside dans la co-appartenance de l'enquêteur et de son interlocuteur à la même temporalité. Cette transformation de tout un univers bien vivant en un monument ethnographique est au centre de la critique qu'ont élaborée Fabian et les anthropologues américains se réclamant du postmodernisme. On aurait affaire à un véritable processus d'aliénation qui prolongerait l'exploitation dont ont fait l'objet les sociétés traditionnellement privilégiées par les études ethnologiques. L'allochronisme est dénoncé comme l'expression intellectuelle d'un projet plus général de domination. Il y aura toujours dans cette conception de l'anthropologie, des temps séparés : " their time " et " our time " qui ne sauraient coïncider. Le décalage postulé entre les temporalités devient une condition indispensable à l'assomption d'une altérité qui entretient le fonds de commerce des anthropologues. Dans ces conditions, la mise en texte de ce qui se présente à l'origine comme interlocution entre eux et nous devient un moment capital de cette transmutation presque magique, au terme de laquelle, est produite une abstraction telle que le Nuer, le Kwakiutl ou le Nambikwara. " L'absence de notre temps de l'Autre, aura été son mode de présence dans nos discours - comme un objet et une victime "24. L'anthropologie est-elle le produit d'un " déni de contemporanéité " (denial of coevalness)? Certains auteurs n'hésitent pas à s'en prendre aux classiques de la discipline. Témoin, l'analyse d'A. Appadurai ${ }^{25}$ consacrée à l'Homo hierarchicus de L. Dumont. La critique porte sur le primat réservé au principe hiérarchique dans l'étude des castes en Inde. Selon Appadurai, le livre de Dumont illustre la propension des études orientalistes à essentialiser leur objet: il y a là une réification de la caste conçue comme l'institution prééminente. En second lieu, Dumont a tendance à " exotiser " son objet. Enfin, la notion de totalisation est au cœur d'une théorie qui fait d'un trait spécifique un phénomène social total. Si la hiérarchie est bien un aspect fondamental de la société indienne, il n'en est pas moins nécessaire de débattre du statut de ce concept. Une fois encore, ce qui est mis en doute, c'est une vision globalisante et péremptoire de la différence culturelle. Celle-ci a l'avantage de la clarté : elle embrasse la multiplicité des données empiriques en leur conférant une signification et une cohérence. Mais on conçoit que cette approche ne satisfasse pas les tenants de la postmodernité. En mettant l'accent sur ce qui distingue la culture de l'observé de celle de l'observateur, Dumont creuse en effet l'écart. La reconnaissance du caractère irréductible de l'altérité devient le moteur de l'entreprise anthropologique. Au prix d'éviter tout débat sur la contemporanéité. Eux et nous : deux mondes sur une même planète. Voilà ce que ne peuvent accepter les postmodernes : leur critique vise précisément la conception idéaliste qui consiste à figer les faits de culture dans des totalités séparées et imperméables. Du temps au texte En mettant en cause les conditions de production de l'Altérité dans le discours anthropologique, la critique va se focaliser de plus en plus sur la textualité. Pour Clifford ${ }^{26}$, par exemple, il s'agit de traquer les stratégies discursives par lesquelles s'affirme l'autorité de l'ethnographe. Ce dernier sujet de l'énonciation s'institue comme étant le garant de la validité de ce qui n'est tout au plus qu'une représentation construite. " J'étais là ", affirme le sujet de l'énonciation, tout en prenant soin de se retirer du texte, au nom de la toute puissance des données objectives. L'auteur se cache, mais l'autorité est bel et bien présente. C'est 
cette dénégation qui sous-tend l'écriture de l'ethnographie. La fausse modestie de l'anthropologue masque mal sa volonté de puissance. A la manière d'un compte rendu d'expérience, la monographie se déploie dans un présent intemporel qui finit par occulter la réalité historique dans laquelle s'est opérée la recherche. Le discours objectiviste de l'anthropologie a un fort pouvoir d'occultation, dans la mesure où il cultive le monologue. Les voix des " observés " sont recouvertes, au nom de la cohérence du projet scientifique. Cette critique du caractère monologique du texte ethnographique n'est pas seulement valable à l'encontre des produits classiques du structuralo-fonctionnalisme ${ }^{\circ}$; elle s'applique aussi à l'anthropologie interprétative de Geertz. Ce dernier, en insistant sur la nécessité de traiter les cultures comme des textes, met à nu le rôle de l'auteur dans l'interprétation et les procédures rhétoriques qu'il met en œuvre. La perspective herméneutique n'en institue pas moins une frontière infranchissable entre l'univers de l'observateur et les cultures qu'il explore. Pour les postmodernistes, il importe de sortir de ce monologisme, d'abolir le clivage entre " eux " et " nous ", en restituant la contemporanéité des échanges discursifs qui caractérisent la pratique de terrain. C'est en réintroduisant les voix qui ont participé à l'élaboration du texte, pour ensuite se voir occultées, au nom de la science, qu'on parviendra à rendre compte du processus d'enquête. Le travail de l'anthropologue qui consiste à recombiner des fragments hétéroclites, à puiser ses données dans des situations souvent disparates doit apparaître dans tout son relief. Ne faut-il pas alors que l'expérience ethnographique s'inscrive dans un texte lui-même expérimental, qui s'invente des formes plus adéquates que le discours neutre de l'autorité savante? On obtiendrait ainsi ces trois caractères de " polyphony, perspectival relativity, fragmentation " que S. Tyler ${ }^{27}$ revendique pour l'écriture de l'anthropologie. Celle-ci s'apparenterait à un art du collage, " un collage de voix pleines, autres que celle de l'ethnographe, autant que d'exemples de preuve 'trouvée', de données incomplètement intégrées au corps de l'interprétation gouvernant l'oeuvre " ${ }^{28}$. Le dialogisme, l'hétéroglossie, offrent le moyen de restituer l'espace polyphonique dans lequel se construit la recherche. Cette stratégie qui vise à redonner place aux échanges discursifs qui ont cours tout au long du travail d'enquête, est surtout pensée en regard d'un paradigme objectiviste, d'un régime de vérité qui est posé comme problématique et politiquement contestable : la mise à distance de l'Autre devient une forme sournoise de domination. On lui préfère une poétique où la complexité, l'ambiguïté, le paradoxe, retrouvent tous leurs droits. C'est une nouvelle politique du texte qui s'esquisse: les notions de poétique et de politique figurent d'ailleurs bel et bien dans le titre de Writing Cultures. L'objectivisme qui préside aux descriptions savantes, l'obsession des données et des faits, a fini par produire des totalités figées, les cultures, dont on il s'agirait d'appréhender la cohérence et l'unité. C'est contre cette représentation que s'insurgent les postmodernes, et la référence à la contemporanéité devient ici stratégique. D'une part, face aux conceptions qui traitent les cultures comme des abstractions, elle permet de réintroduire l'histoire et la complexité du réel; d'autre part, s'impose l'idée que le présent d'une culture est un patchwork où viennent se croiser les significations et les pratiques les plus hétéroclites. Clifford ${ }^{29}$ décrit l'univers de cette fin de siècle comme " Un véritable espace global de connections culturelles et de dissolutions est devenu imaginable: l'authenticité locale se rencontre et émerge dans des formations... transitoires ". Dans ce contexte, la notion d'altérité se dilue : où commence la culture de l'observateur? Où s'arrête celle de l'observé? En fait, l'anthropologie devient une pratique cosmopolite, en prenant acte d'un phénomène de 
grande ampleur : la mondialisation. Selon l'expression imagée de P. Rabinow ${ }^{30}$, la figure $\mathrm{du}$ chercheur en cette fin de siècle est celle du " cosmopolitan amateur ". Il combine tout à la fois une intelligibilité des interdépendances qui caractérisent notre planète et la conscience des singularités, des histoires et des destins ${ }^{31}$. Bien sûr, le local est longtemps demeuré l'un des points d'ancrage d'une démarche qui s'est toujours définie par son rapport au terrain. Mais que signifie aujourd'hui cette référence au terrain, dans un contexte marqué par un processus très général de déterritorialisation qu'illustrent la circulation des hommes et des produits à l'échelle mondiale, dans un processus qui ne cesse d'articuler et de recomposer les différences culturelles? Les représentations complexes qui s'élaborent dans le contexte de ces déplacements, les " global ethnoscapes "32, méritent d'être examinées de près par les anthropologues. " La représentation de la différence ne doit pas être lue à la hâte, comme le reflet de traits ethniques et culturels 'pré-donnés', entablés dans la tradition. L'articulation sociale de la différence, dans la perspective de la minorité, est une négociation toujours en cours, complexe, qui cherche à autoriser des hybridités culturelles émergentes dans un moment de transformation historique "33. La conception de l'altérité qui a présidé au développement de l'anthropologie est devenue anachronique. Dans un monde ouvert où circulent non seulement les individus et les groupes, mais l'information sous toutes ses formes, les anthropologues ont affaire à des expériences de plus en plus composites. Dans ces conditions, l'assignation d'une différence radicale entre le temps de l'observateur et celui de l'observé fait figure d'obstacle épistémologique. Il s'agit de se donner les moyens de reproduire dans le texte leur contemporanéité. Mais le " semiotic deconstruct turn " (Rabinow) qui caractérise cette démarche, s'il a bien un pouvoir critique à l'égard d'un regard éloigné qui crée une distance infranchissable par rapport à l'objet de l'anthropologie, reste curieusement discret sur la nature même de cette (post)modernité qui constitue l'unique et englobant environnement des sociétés sur la planète. La question du régime d'historicité sous lequel nous vivons aujourd'hui ne saurait cependant être éludée. La critique des anthropologues " déconstructionnistes " ne semble pas très intéressée par le temps : elle ne déploie pas toutes les conséquences de l'analyse du postmodernisme telle que la développe par exemple Jameson. Selon ce dernier, le postmodernisme n'est pas " a style, but rather as a cultural dominant "34. Il se présente, en réaction au modernisme et à ses avant-gardes (Picasso, Joyce, et autres), non comme porteur de nouveauté, mais comme déconstruction de l'expression. La parodie, le pastiche, sont au cœur d'un jeu où le présent s'annexe le passé, où l'on joue à copier les chefs-d'œuvre en en détournant le sens, où l'on réifie, où l'on instrumentalise des produits culturels sans nullement se préoccuper d'aller de l'avant. Le postmodernisme, c'est d'une certaine manière la fin de l'histoire; ou plutôt, le triomphe de l'historicisme. Par historicisme, Jameson entend ici, à l'inverse d'un retour au passé, une prolifération de références, non au passé mais à nos représentations du passé, tels ces films " rétro " qui mettent en représentation nos représentations du passé, ou un ensemble architectural, tel l'hôtel Bonaventura construit par John Portman à Los Angeles. La réinscription du futur dans un horizon sans progrès Du point de vue qui nous intéresse, celui de l'histoire et du temps, le postmodernisme pose une autre question qui a trait à l'essence même de la séquence temporelle : présent, passé, futur. Sans évoquer explicitement une fin de l'histoire : on en est au va-et-vient entre passé et présent. Mais sans se situer dans l'horizon du futur. La notion même de " post " remet en cause l'idée d'un sens unique du temps. On est à mille lieux de l'idée de perfectionnement (Rousseau) qui apparaissait consubstantielle aux représentations de 
la modernité. Le postmodernisme correspond dans une certaine mesure à la mise en spectacle de l'effondrement de l'idée régulatrice de progrès qui a longtemps orienté les comportements et les rapports sociaux. Le triomphe du postmodernisme traduit-il la prodigieuse expansion du capital au niveau planétaire, comme le suggère Jameson ? En tout cas, on voit s'imposer une figure du temps où le futur ne s'identifie plus au progrès, où s'opère sans cesse une mise en abîme du présent. On pourrait alors envisager le postmodernisme comme la thématisation d'un certain rapport au temps qui ne serait plus le même que celui qui a marqué des époques antérieures. Cette perspective peut sembler plus intéressante que celles que privilégient les anthropologues déconstructionnistes. Ces derniers ont tendance à rester enfermés dans une vision du temps qui ne s'éloigne guère des présupposés qu'ils critiquent. S'ils mettent en cause le décalage artificiellement créé entre Soi et l'Autre, ils n'en acceptent pas moins l'orientation, implicite et prégnante chez les anthropologues, qui privilégie comme axe temporel de leur recherche, la séquence passé-présent. Certes, la dénonciation de l'allochronisme marque bien le souci d'échapper à " l'illusion archaïque " que traquait autrefois Lévi-Strauss. Surmonter le grand partage, c'est-àdire ne plus rejeter l'altérité dans un univers indexé sur la tradition, arrimé au passé, englué dans ses origines : tel est bien le projet des anthropologues critiques. Mais ils nous livrent peu d'instruments pour penser le contemporain, au-delà de l'opposition simple entre modernité et tradition. Et surtout, ils semblent se désintéresser de la question, incontournable dans la réflexion sur le postmodernisme, du régime de temporalité dans lequel s'inscrit notre présent. Est-ce à force de " déployer les sociétés humaines dans l'espace ", que l'anthropologie en est arrivée à ignorer à ce point le type de relation au temps constitutif de ce qu'elle désigne sous les notions de contemporain et de (post)moderne? En prenant pour objet l'Europe politique, on est cependant confronté en permanence à la question du temps. Le thème de la durée est très présent dans cet univers. Ainsi, revient souvent dans le discours de mes interlocuteurs l'idée que la Commission est une institution jeune. Par contraste avec les administrations nationales, la Commission est décrite par ses agents comme un organisme qui, en un temps très limité (la CECA date de 1950), s'est progressivement étoffé et transformé, en liaison avec les objectifs fixés par les traités européens successifs et avec l'intégration de nouveaux Etats membres. L'ancrage historique de l'institution ne constitue pas une réelle préoccupation. Ce qui frappe, en revanche, c'est la toute-puissance du présent. Un mot résume cette attitude : le vocable " construction " toujours associé à l'Europe. Celle-ci n'en finit pas de s'édifier ${ }^{\circ}$ l'inachèvement est en quelque sorte la propriété maitresse du processus communautaire. On parle sans cesse de conclure, qu'il s'agisse des dossiers en cours, des négociations en suspens, de l'élargissement qui se profile. Mais, dans cette forme politique inédite que constituent les institutions européennes, mélange d'inter-gouvernemental et de supra-national ${ }^{35}$, l'achèvement est remis à plus tard: l'urgence s'interpose devant l'idéal toujours reporté. Tout se passe en effet comme si la Communauté était condamnée à vivre perpétuellement dans la fuite en avant. Elle est le bras séculier d'un projet dont l'achèvement est toujours reporté. $\mathrm{Au}$ processus de déterritorialisation correspond un mouvement de déshistoricisation. Dans les groupes classiquement étudiés par les anthropologues européanistes, la tradition joue un rôle fondamental. Elle opère comme un fil conducteur qui oriente les destins individuels. Ces sociétés ont une " mémoire longue " ${ }^{36}$ : cette mémoire est le contrepoint omniprésent de l'activité quotidienne. En témoignent l'importance des généalogies et le prestige accordé à l'ancestralité, les élaborations symboliques et les 
mythes célébrant les hauts faits des pionniers. Quand des bouleversements interviennent au sein de la communauté, c'est une fois encore en se tournant vers le passé qu'on se donne le moyen de penser les aléas du présent. La tradition balise l'actualité ; elle lui confère un sens et offre la possibilité de maîtriser dans une certaine mesure la contingence de l'événement. Loin d'être figée, elle est l'objet d'une invention permanente, comme en témoignent éloquemment les modifications que subissent tout au long du temps les symboles et les rituels. Cette dialectique du présent, de la mémoire et de la tradition constitue à elle seule un objet anthropologique fascinant. Le fait qu'elle soit introuvable dans les représentations des praticiens de l'Europe mérite d'être souligné. L'on ne cesse en effet d'évoquer la " construction " européenne: la Communauté est vécue par ses artisans comme un chantier. On n'imagine même pas que cette construction puisse un jour être terminée. " L'Europe ne se fera pas d'un coup ni dans une construction d'ensemble: elle se fera par des réalisations concrètes créant d'abord une solidarité de fait " déclarait Robert Schuman, en présentant le projet de Communauté européenne du charbon et de l'acier ${ }^{37}$. Commentant cette déclaration, Jean Monnet a écrit : " C'était le choix fondamental d'une méthode à intégrer sans fin les choses et les esprits. " ${ }^{38}$ L'idée d'impliquer et d'imbriquer les Etats dans un système d'interdépendances toujours plus poussé s'est concrétisée dans la multiplication des directives qui s'articulent les unes aux autres selon une logique de plus en plus implacable. Il s'agit bien là d'un processus quasi mécanique, présenté aux ressortissants européens comme irréversible. C'est la perspective de l'avenir qui donne un sens à ce processus : alors seulement, l'Union européenne aura trouvé son accomplissement, et l'intégration se trouvera enfin réalisée. Mais cet horizon demeure lointain et indéterminé. A la différence des Etats existants, la Communauté se vit elle-même comme un processus dynamique tendant vers un but qu'elle est encore loin d'avoir atteint. Cet inachèvement est une donnée essentielle pour qui veut comprendre le mode de fonctionnement des institutions européennes. Il implique en effet l'absence de référentiel stable, et, en pointillé, la perspective toujours ouverte d'une montée en puissance de cette construction dont on ne sait trop quelle sera la configuration définitive. La représentation du temps de l'Europe communautaire est donc totalement différente de celle qui prévaut dans les communautés traditionnelles. Elle est tout entière orientée vers le futur. C'est ce qui permet aux artisans de l'Europe de résister aux mouvements de reflux qu'on observe périodiquement. Les cinquante dernières années se présentent en effet comme une succession d'accélérations significatives, avec le plan Schuman, les traités de Rome, les intégrations de nouveaux Etats membres, la signature de l'Acte unique et le traité de Maastricht. Entre chacune de ces avancées se produisent des périodes de blocage. La machine semble grippée, on sombre dans l'europessimisme, voire l'euroscepticisme. Au sein du Parlement, dans les services de la Commission, ce type d'alternance est parfois difficile à assumer. Il faut vivre au jour le jour les à-coups de l'Europe. L'invocation de l'avenir offre le moyen le plus efficace d'assumer cette situation. L'Europe est vécue comme un projet dont l'échéance est toujours reportée. L'action des fonctionnaires et des élus communautaires est guidée par cette assomption du futur. Tous insistent sur le fait que la situation n'est pas figée : même les institutions dans lesquelles ils travaillent peuvent encore connaître des changements significatifs. A la différence des Parlements nationaux, l'Assemblée européenne n'a pas encore des attributions stables et bien définies. L'apparition de nouvelles procédures (coopération, codécision) lui a permis d'étendre son champ de compétences, et l'horizon reste ouvert. Le rôle de la Commission fait aujourd'hui l'objet 
de discussions et, là aussi, une évolution est probable. L'avenir institutionnel de l'Union demeure très ouvert; de même, il règne une grande incertitude, quant aux frontières définitives que se donnera la Communauté. L'élargissement à quinze apparaît déjà comme le prélude à une nouvelle extension vers l'Est. L'appartenance communautaire se vit donc sur le mode de l'inachèvement. La toute-puissance du présent, l'invocation insistante du futur sont au cœur des représentations quotidiennes des praticiens de l'Europe. A la Commission, on aime travailler dans l'urgence; cette organisation est particulièrement performante quand elle se voit assigner des objectifs à court terme. Le mot " finaliser " revient souvent dans le vocabulaire des fonctionnaires. Qu'il s'agisse d'un dossier, d'une réunion, d'une négociation en cours, il importe de terminer dans les délais, il faut que l'action entreprise se réalise. La Commission n'est jamais aussi l'aise que dans les périodes où le rythme s'accélère. Les différentes instances européennes travaillent en permanence sous la pression du calendrier. C'est d'ailleurs sous le signe du calendrier que s'est placée la Commission quand elle proposa l'Acte unique : l'aspect le plus spectaculaire de cette initiative consistait en effet à fixer une date limite pour la réalisation du grand marché européen. Un régime d'historicité Ainsi se construit la Communauté, dans une dynamique qui lui est propre. L'important, c'est d'aller de l'avant, sans se retourner, vers un avenir qui donnera son sens définitif au travail réalisé. " On conduit sans rétroviseur ", explique un haut fonctionnaire. Dans les périodes de reflux, l'atmosphère s'assombrit : on observe au sein de la Commission un courant d'incertitude et même d'angoisse. L'absence de référent fort, tel celui que représente, pour des fonctionnaires nationaux, l'Etat, ses règles, son gouvernement, devient palpable. Ce qui transparaît alors, c'est l'incapacité de la Communauté à faire retour sur elle-même. On mesure dans ces conjonctures, la difficulté qu'éprouvent les uns et les autres à se situer par rapport à une histoire. Tout se passe comme si l'Europe devait se réinventer chaque jour, affirmer une éternelle jeunesse. On semble ignorer le travail de la mémoire, si bien que chaque crise successive se trouve neutralisée par une chape d'oubli. La référence au passé se limite à une brève évocation des pères fondateurs; aucun signe ne vient témoigner de la présence d'une tradition. Toute référence à cette dernière semble incongrue dans un tel contexte. Ce déficit de tradition, l'absence de réflexivité par rapport au passé caractérisent un certain rapport du fonctionnement institutionnel européen au temps. Dans cet univers de compromis, la mémoire ne semble pas avoir sa place, et l'on peut voir comme une allergie à dans le cette sorte de déficit symbolique et rituel. On insistera aussi sur l'importance de la dialectique entre présent et futur qui modèle le devenir de l'Europe politique. Une analyse rigoureuse ne saurait sous-estimer l'importance prise ici par la dimension du virtuel. Le processus politique s'opère sous le signe d'une double indétermination quant à sa forme définitive (une nouvelle espèce d'Etat-Nation, une fédération d'Etats, une structure postnationale ?) et quant au nombre des participants impliqués (où s'arrêtera l'élargissement ?). On a donc affaire à un dispositif qui produit des effets politiques massifs, mais qui demeure, à proprement parler, innommable et indéfini. L'introduction du concept (lui-même encore flou) de gouvernance témoigne bien de la difficulté à appréhender tout à la fois le lieu du pouvoir européen et l'unité d'un projet qui l'orienterait. On se situe dans l'émergent, dans le virtuel de ce qui sera peut-être un jour une réalité politique globale et unifiée. La présence permanente d'un champ de problèmes ouvert, qui inclut désormais tout un discours sur " l'absence de réponse aux problèmes ", est révélatrice. La dimension du virtuel et le rapport au temps qu'elle institue nous écarte définitivement de la vision longtemps prédominante du progrès. 
Elle nous oblige à réfléchir sur ce qu'on nous permettra, faute de mieux, de désigner comme la " temporalisation " contemporaine du politique. On se réfère à une notion introduite par le philosophe R. Koselleck. Ce dernier s'est en effet penché sur l'évolution de la pensée du temps depuis le Moyen Age. Il montre notamment comment la naissance de l'Etat moderne s'est accompagnée de ce qu'il nomme une " laïcisation du temps " : jusqu'au milieu du XVIe siècle, la représentation du temps intègre le futur, en l'espèce, la fin des temps. L'histoire de l'Eglise prend en compte la fin possible du monde ; la notion de salut y joue un rôle primordial. Elle est l'horizon d'attente dans la perspective duquel se déroule l'histoire de l'Eglise. Dans cette représentation du temps, il est clair que le futur se trouve intégré au temps, il joue un rôle intégrateur essentiel dans la pérennisation de la souveraineté de l'Eglise Romaine. On est aux antipodes d'un temps linéaire, puisque la fin du monde se trouve en quelque sorte inscrite dans l'expérience immédiate du présent et du passé. La rupture des Temps modernes se produit avec l'émergence d'une histoire proprement humaine, déconnectée de l'attente d'une fin du monde. Si le Saint Empire romain germanique s'inscrit dans une eschatologie dont il tire légitimité et qui lui assigne son rôle, l'Etat moderne apparaît désormais sans lien avec l'histoire sainte et capable de dompter les factions religieuses. Dans ce contexte, la fonction prophétique se trouve battue en brèche. C'en est fini d'une conception qui renvoyait à la certitude du Jugement Dernier, essentielle dans l'établissement des principes de l'action. On entre dans un univers où le futur est pensé sur le mode de la probabilité et laisse place aux calculs et aux stratégies. Il s'agit de calculer la probabilité d'événements susceptibles d'arriver ou non: le pronostic se substitue à la prophétie. On passe d'une structure temporelle statique, caractérisée par la cyclicité de l'histoire, à une structure dynamique où règnent l'accélération et l'irréversibilité du temps. L'avenir est désormais synonyme d'inconnu; le fait qu'il fonde sur nous de plus en plus vite, implique que les praticiens du politique adoptent une attitude bien différente des comportements antérieurs marqués par une attente eschatologique. On rattrape la fuite du temps grâce à la science du pronostic, devenue un instrument indispensable pour agir sur une situation politique. Celle-ci intègre dans son calcul les trois dimensions du temps. Pour maîtriser le présent, il faut être en mesure d'anticiper le futur en se fondant sur les enseignements du passé. Koselleck fait bien apparaître à quel point la temporalisation de l'histoire se transforme à partir des Temps modernes. Il thématise cette mutation en introduisant les concepts de champs d'expérience et d'horizon d'attente. La grande nouveauté de la fin du XVIe siècle, c'est la dissociation entre attente et expérience Depuis lors, " non seulement le fossé entre le présent et le futur ne cesse de s'agrandir mais il nous faut toujours à nouveau surmonter la différence entre l'expérience et l'attente et ce, de façon toujours plus rapide, pour pouvoir vivre et agir "39. La temporalisation moderne correspond à la tension permanente entre attente et expérience inséparable de la notion de progrès. L'intérêt de la démarche de Koselleck tient à son souci de problématiser la représentation du rapport entre présent et futur qui a dominé l'âge moderne. Aujourd'hui cependant la situation est bien différente : la conception qu'il analyse, dominée par l'idée de progrès, a laissé place dans le champ politique à une configuration de pratiques et de discours qui dessinent un horizon d'incertitude. La visée du progrès s'inscrivait dans la tension entre présent et futur et travaillait à combler le fossé entre expérience et attente. Ainsi, a-t-on construit des " concepts d'attente " dans le but d'anticiper le mouvement futur de l'histoire : républicanisme, socialisme, tous les " ismes " qu'on retrouve dans le discours politique, mais aussi au 
cœur des sciences sociales, participent de cette entreprise. Or, désormais, cette vision des choses est remise en cause dans des domaines aussi différents que la science et la politique, mais qui ont pour point commun de traiter du futur. Certes, les sciences vont de l'avant, mais, depuis longtemps déjà, elles mettent en question la positivité du progrès ; de même, en politique, le doute s'est-il institué à propos des " lendemains qui chantent " et l'impact du changement : le mot " révolution " a été banni du vocabulaire politique. Mais surtout, ce que révèlent l'histoire et l'anthropologie de la construction européenne, c'est l'existence d'un nouveau mode de temporalisation. C'en est fini de la tension entre attente et expérience, pour reprendre les concepts de Koselleck. La temporalisation postmoderne réintègre le futur dans le présent. On a vu à quel point cette présence du futur est un élément constitutif du discours et des représentations associés à l'Europe. Le dispositif idéel produit dans l'univers communautaire nous installe directement dans le virtuel. La toute-puissance de l'agenda et du calendrier imposent comme déjà présents des événements non encore advenus: ainsi de la monnaie unique ou de l'élargissement aux pays de l'Est. La situation n'est pas sans analogie dans le domaine des sciences où, pour citer un exemple récent, le clonage fait désormais partie de l'actualité, bien avant que l'expérience ait été tentée sur les humains. Ce qui compte, c'est que la présence du futur, modèle et contextualise l'expérience du présent. On comprend mieux pourquoi la question de la forme politique définitive de l'Europe est toujours reportée. C'est que la puissance du dispositif réside dans le mélange qu'il réalise entre le virtuel et le réel. Le virtuel est un carburant beaucoup plus efficace que la production d'un ordre politique qui figerait les relations et pourrait susciter des réactions de rejet difficilement contrôlables : on connaît bien les réticences de la plupart des Etats membres à aliéner tout ou partie de leur souveraineté. Non identifié, l'objet politique européen trouve son efficacité optimale. Il détermine les évolutions nationales, il en infléchit la donne, sans pour autant être, en tant que tel, affecté par les réactions qu'il engendre. Ce n'est pas un paradoxe que de conclure que l'omniprésence et la performance de l'Europe politique ont partie liée avec les dimensions du virtuel et de l'incertitude qui la caractérisent. Pour l'anthropologie et les autres sciences sociales, cette situation peut sembler très énigmatique. Héritières du XIXe siècle, elles sont tributaires de la conception du temps décrite par Koselleck, marquée par la disjonction entre présent et futur, même si la sociologie s'est plutôt préoccupée de la relation entre ces deux séquences temporelles, alors que, on l'a vu, l'anthropologie a surtout cherché à articuler présent et passé. Les sciences sociales n'en sont pas moins restées engluées dans cette tension entre expérience et attente ; elles ont été grandes consommatrices d'" ismes " : elles en usent encore pour qualifier le projet européen, comme en témoigne notamment la référence au fédéralisme. Pour en revenir à l'anthropologie, constatons que l'étude de la construction européenne l'amène non seulement à repenser la question de l'identité au travers d'une approche des " régimes d'altérité ", mais aussi à prendre au sérieux la problématique du temps, généralement assimilée à la problématique des rapports entre ethnologie et histoire. Dans cette perspective, l'anthropologie du présent et du contemporain ne peut éluder la réflexion sur la temporalisation. L'intrication du futur et du présent, du virtuel et du réel est ainsi apparue comme un élément constitutif du processus politique communautaire. Afin de rendre compte de cette complexité, l'anthropologue doit affiner ses instruments conceptuels, car c'est au prix d'une analyse attentive aux représentations du temps qu'on peut tenter de mieux comprendre ce qui est aujourd'hui en jeu dans la construction européenne. 


\section{NOTES}

1. Directeur du LAIOS-CNRS.

2. Abélès M., La Vie quotidienne au Parlement européen, Paris, Hachette, 1992 ; En Attente d'Europe, Paris, Hachette, 1996.

3. Abélès M., Jours tranquilles en 89. Ethnologie politique d'un département français, Paris, Odile Jacob, 1989.

4. Augé M., " Qui est l'autre ? Un itinéraire anthropologique ", L'Homme, 103, 1987, pp. 7-26.

5. Marcus G., Fischer M., Anthropology as cultural critique, University of Chicago Press, 1986.

6. Lévi-Strauss C., Anthropologie structurale deux, Paris, Plon, 1973, p. 39.

7. Ibid., p. 26.

8. Ibid., p. 40.

9. Lévi-Strauss C., La Pensée sauvage, Paris, Plon, 1961, p. 339.

10. Balandier G., Anthropo-logiques, Paris, PUF, 1974, p. 205.

11. Godelier M., Horizons, trajets marxistes en anthropologie, Paris, Maspéro, 1973.

12. Terray E., Le marxisme devant les sociétés " primitives ", Paris, Maspéro, 1969.

13. Pouillon, " Lévi-Strauss ", Dictionnaire de l'ethnologie et de l'anthropologie, Paris, PUF, 1992, p. 418.

14. Ortner S., " Theory in Anthropology since the Sixties ", Comparative Studies in Society and History, 26, 1, 1984, pp. 126-166.

15. Bourdieu P., Esquisse d'une théorie de la pratique, Genève, Droz, 1972.

16. Sahlins M., Historical Metaphors and Mythical Realities : Structure in the Early History of the Sandwich Islands Kingdom, Ann Arbor, University of Michigan Press, 1981.

17. Bensa A., " De la micro-histoire vers une anthropologie critique " in Revel J. (dir.), Jeux d'échelles, Paris, Hautes Etudes, Gallimard/Le Seuil, 1996, pp. 37-70.

18. Levi G., Le pouvoir au village, Paris, Gallimard, 1989, p. 41.

19. Ibid., p. 15.

20. Fabian J., Time and the Other. How Anthropology Makes its Object, New York, Columbia University Press, 1983.

21. Fabian, op. cit..

22. Bensa A., op. cit., p. 49.

23. Fabian J., op. cit., p. 154.

24. Ibid., p. 154.

25. Appadurai A. in Marcus G. (ed), Rereading Cultural Anthropology, Durham and London, Duke University Press, 1992.

26. Clifford J., Writing Culture : the Poetics and Politics of Ethnography, University of California Press, 1986.

27. Tyler S., 1984, p. 137.

28. Clifford J. and Marcus G. (eds), The Predicament of Culture, Harvard University Press, 1988, p. 147.

29. Ibid., 1988, p. 4.

30. Cf. Essays on the Anthropology of Reason, Princeton, Princeton Studies in Culture/ Power/History, 1996, p. 24. 
31. Ibid., 56.

32. Appadurai A. in Marcus G., 1992, op. cit..

33. Bhabha O.K., The Location of Culture, London and New York, Routledge, 1994, p. 2.

34. Jameson F., " Postmodernism or the Cultural Logic of Left Capitalism ", New Left Review, 146, 1984, pp. 53-92.

35. Cf. l'analyse de J.L. Quermonne (1993) qui montre la coexistence permanente au sein des institutions européennes de deux stratégies, l'une axée sur la coopération intergouvernementale, l'autre mettant en oeuvre le principe de supranationalité.

36. Zonabend F., La mémoire longue, Paris, PUF, 1979.

37. Déclaration du 9 mai 1950.

38. Monnet J., Mémoires, Paris, Fayard, 1976, p. 434.

39. Koselleck R., Le Futur passé. Contribution à la sémantique des temps historiques, 1979, trad. fr., Paris, Editions de l'EHESS, 1990, p. 322.

INDEX

Index géographique : Bruxelles, Europe, Union européenne

Mots-clés : anthropologie, régionalisme et régionalisation, construction européenne, politique 